\title{
Electromagnetic Risk Analysis for EMI Impact on Functional Safety with Probabilistic Graphical Models and Fuzzy Logic
}

\author{
Lokesh Devaraj, Alastair R. Ruddle, Senior Member, IEEE, and Alistair P. Duffy, Fellow, IEEE
}

\begin{abstract}
For the functional safety of complex systems such as road vehicles, sufficient immunity of the safety-related electronic, electrical and programmable electronic systems to the anticipated electromagnetic environment is required. Current rule-based practices follow established standards for testing the immunity and emission performance at vehicle and sub-system levels. Nonetheless, with increasingly rapid technological changes, confidence may not be sufficient even if the immunity test levels or the number of tests is increased. A risk-based EMC approach, on the other hand, aims to identify the safety hazards due to electromagnetic disturbances and mitigate the associated risks to achieve the required confidence level. Traditional tools used for risk analysis (such as fault tree analysis, event tree analysis and failure mode and effect analysis) may not be sufficient as the complexity level increases. In this paper, a graphical approach is proposed to enable system visualization as well as supporting a comprehensive risk analysis. The possible implementation of a system-level analysis is illustrated with several methods (e.g. Bayesian networks, Markov random fields and fuzzy set theory). These methods could be used to include EM risk factors such as spatial location, functional dependence etc. in order to estimate the risks associated with EMI-related hazards.
\end{abstract}

Index Terms-EMI, functional safety, risk analysis, Bayesian network, Markov random field, fuzzy logic

\section{INTRODUCTION}

With the increasing complexity of road vehicles, the range and number of hazards which could compromise the safety of road users is also increasing. This is evident with the significant rise in the number of vehicles recalled and redesigned by vehicle manufacturers each year [1]. Electromagnetic disturbances (EMDs) from on-board sources (such as noisy components within the vehicle, or passengers' personal devices) as well as off-board external threats (e.g. broadcast transmissions, airport radar systems and nearby transmitters) are

The research leading to these results has received funding from the European Union's Horizon 2020 research and innovation programme under the Marie Skłodowska-Curie grant agreement No 812790 (MSCA-ETN PETER). This publication reflects only the authors' view, exempting the European Union from any liability. Project website: http://etn-peter.eu/.

Both Lokesh Devaraj and Alastair Ruddle are with HORIBA MIRA Limited, Nuneaton, CV10 0TU, UK (e-mail: forename.surname@horibamira.com).

Alistair Duffy is with De Montfort University, The Gateway, Leicester, LE1 9BH, UK (e-mail: apd@dmu.ac.uk).

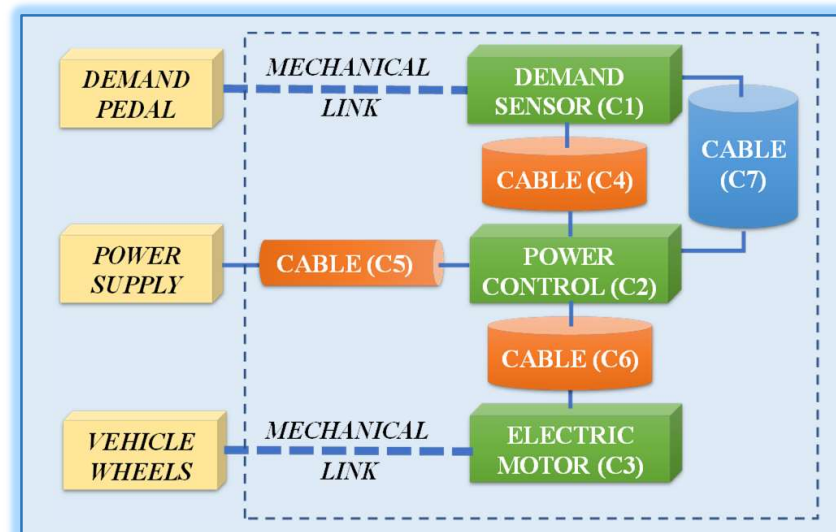

Propulsion system architecture considered for the case study. The sensors, actuators, and power control and distribution module (green), as well as the power cables (orange) and signal cables (blue) form the system of interest.

prominent threats that electromagnetic compatibility (EMC) test standards [2] aim to address. Nonetheless, EMC issues have certainly been responsible for several significant recalls [1].

Functional safety and EMC are two different, but related, system attributes. The latter concerns the ability of a system to function acceptably in its electromagnetic (EM) environment, whereas the former aims to limit the safety risks associated with malfunctions of the electrical, electronic, programmable electronic components to acceptable levels. Functional safety is addressed using a risk-based approach requiring hazard and risk analysis of the specific system, whereas EMC employs a rule-based approach requiring compliance with standardized tests. As electromagnetic interference (EMI) is a potential cause of functional safety issues it is considered that there is a need to achieve a greater alignment between these domains (e.g. [3]-[4]). Currently, however, there is no established methodology to estimate, collectively, the EM risks to system functional safety.

Take-Home Messages:

- EMC and functional safety are generally considered separately, but have overlapping aspects (EM threats to safety-related functions) that need to be better aligned.

- The impact of EMI on individual system functions, plus the additional impact due to functional dependence on other system components which are susceptible to EMI, need to be considered when estimating the safety risks.

- Exhaustive testing of EMI threats is not practicable, but probabilistic graphical models and fuzzy logic methods could facilitate more comprehensive EM risk analyses. 
Accepted version: L. Devaraj, A. R. Ruddle and A. P. Duffy, "Electromagnetic Risk Analysis for EMI Impact on Functional Safety with Probabilistic Graphical Models and Fuzzy Logic," in IEEE Letters on Electromagnetic Compatibility Practice and Applications, doi: 10.1109/LEMCPA.2020.3017483. (C) 2020 IEEE https://ieeexplore.iee.org/document/9171307

Replacing the current rule-based EMC approach with a risk-based approach will require a comprehensive risk analysis of the system. The graphical approach proposed in [5] to help align the various aspects of EMC and functional safety needs to be further extended to be able to assist the estimation of risk parameters (likelihood and consequence of EM impact). In this paper, the risk factors that should be considered for the EM risk analysis are given in Section II. This is followed by a case study for a very simple vehicle propulsion subsystem (as shown in the visual summary above), in order to explain the use of a graphical model to include the risk factors.

In Section III, the possible use of probabilistic graphical models including Bayesian networks (BN) and Markov random fields (MRF), as well as fuzzy set theory, are illustrated. It is considered that a combination of these methods could be used to support the estimation of the system-level EM risks in complex systems.

\section{GRAPHICAL MODEL FOR RISK ANALYSIS}

\section{A. Steps in EMI Risk Analysis}

Any EMD in the system environment is a potential threat that may lead to EMI with undesirable safety consequences. Hence, EM risk analysis consists of two main steps; identification of potential hazards due to EMI, and estimation of their associated risks.

In the automotive industry, functional safety is addressed using ISO 26262 [6]. A core element of this approach is the hazard and risk assessment, which includes the identification of potential causes of functional failures that could adversely affect safety-related systems, which may include EMI. Currently, it is assumed that compliance with vehicle immunity standards will ensure that such EMI issues for functional safety are adequately addressed. However, the increasingly rapid pace of technological change makes it difficult for EMC standards to keep pace with these developments. Thus, it is becoming increasingly important to be able to undertake more comprehensive analysis of potential EMI-related risks.

To estimate the EMI risks associated with the identified hazards, it is necessary to determine the likelihood of EMI and its impact on the safety-related system functions, as the risk is a combination of threat likelihood/probability and the severity of impact. For complex systems, the correct functioning of multiple components is usually required to perform a system function as intended. Hence, it is important to take in to account all EM risk factors, such as those given in Table 1, when estimating the system-level risks to safety functions. Although EMI effects inherently systematic they may also manifest as pseudo-random, in that both the threat and the vulnerable state, which are potentially time-varying, must be coincident for an effect to occur. Furthermore, ageing, corrosion and variations in manufacturing and materials introduce further probabilistic aspects.

The graphical approach introduced in [5] consists of nodes to represent the physical components of a system, and arcs/arrows connecting them to represent the functional dependencies/interactions between components (see Fig. 1). The interface cables, which are used for the transmission of electrical signals and power between the components, are also considered as nodes in the graphical model. In addition to the functional dependence, a graphical model can also facilitate the inclusion of other risk factors by assigning each node with suitable variables (e.g. assigning each node with local threat field levels representing the coupling from an external source to their specific spatial locations) corresponding to each of the risk factors
Table 1: The list of component level risk factors to be considered to determine the risk parameters for a system-level EM risk analysis.

\begin{tabular}{ll}
\hline \hline EM Risk Factors & Risk Parameters \\
\hline $\begin{array}{l}\text { Coupling paths (conducted and radiated) } \\
\text { Spatial location of safety-related components } \\
\text { within the system }\end{array}$ & $\begin{array}{l}\text { Likelihood of EMI } \\
\text { Likelihood and impact } \\
\text { of EMI }\end{array}$ \\
$\begin{array}{l}\text { components dependence/interactions with other } \\
\text { Change in EM properties with ageing, corrosion } \\
\text { and other environmental effects }\end{array}$ & $\begin{array}{l}\text { Impact of EMI } \\
\text { of EMI }\end{array}$ \\
\hline
\end{tabular}

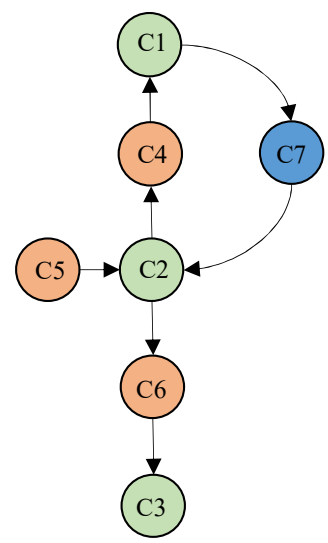

Fig. 1. Graphical model of the system for the propulsion system given in visual summary.

given in Table 1. Risk parameters related to factors like ageing and corrosion can be time-dependent variables. The nodes could also be further enriched with information about their spatial location in the physical implementation and characteristics as a source or victim. The spatial location of components may change before the final design is agreed. Hence, the node variables of the graphical model would also need to be dynamically changed during the risk analysis.

\section{B. Case Study: Propulsion System}

A graphical model for the propulsion system architecture given in the visual summary consists of nodes representing a sensor $\mathrm{C} 1$, a controller $\mathrm{C} 2$, an actuator $\mathrm{C} 3$, and interface cables $\mathrm{C} 4-\mathrm{C} 7$ as shown in Fig. 1. Since, EMI is a common cause for functional deviation, there is a likelihood that more than one component involved in a system function are affected. In such cases, the degree of impact can be much higher, which can be identified in the early stages of system development by considering functional dependence as a risk factor in the proposed graphical model. Functional dependence is mapped in the graphical model with arrows, which can be useful when estimating the overall risk to system functions that are carried out by multiple functions. For instance, the arcs that terminate on node $\mathrm{C} 2$, originating from nodes $\mathrm{C} 5$ and $\mathrm{C} 7$, represent the functional dependence of the power controller on electrical power and demand data delivered via the respective cables. Such dependencies on other components will change the degree of EMI impact and, hence, the severity of the consequence. Determining the functional deviation due to EMI is also very difficult, since each component function might have several functional states (for example, if we consider $\mathrm{C} 7$ to deliver a 6-bit code to $\mathrm{C} 2$, there are $2^{6}$ possible ways of EMI having an impact on $\mathrm{C} 7$, each with varying degree). 
Accepted version: L. Devaraj, A. R. Ruddle and A. P. Duffy, "Electromagnetic Risk Analysis for EMI Impact on Functional Safety with Probabilistic Graphical Models and Fuzzy Logic," in IEEE Letters on Electromagnetic Compatibility Practice and Applications, doi: 10.1109/LEMCPA.2020.3017483. (C) 2020 IEEE https://ieeexplore.iee.org/document/9171307

For a comprehensive risk analysis, the likelihood of intolerable functional deviation is required. Methods that are discussed in the next section could be used combine limited data and expert judgement in order to estimate the overall likelihood at system level.

\section{METHODS FOR RISK ANALYSIS}

\section{A. Bayesian Networks}

$\mathrm{BN}$ are directed acyclic graphs, with nodes representing any variable, while the arcs (arrows) inter-connecting pairs of nodes specify the conditional dependence between them in a probabilistic, deterministic or functional sense. In a $\mathrm{BN}$, each node is connected to at least one other node. If $(u, v)$ is an arc from node $u$ to node $v$, then the node $u$ is a parent of $v$ and this is denoted as $u \in \mathrm{pa}(v)$, where, $\mathrm{pa}(v)$ denotes the set of parents of node $v$ in the graph. For example, in the graph shown in Fig. 2(a), the direction of the connecting arc between $\mathrm{N} 1$ and $\mathrm{N} 2$ indicates that $\mathrm{N} 1$ is a parent of N2, and the set of parent nodes of $\mathrm{N} 2$ is given as $\mathrm{pa}(\mathrm{N} 2)=\{\mathrm{N} 1, \mathrm{~N} 3\}$.

The $\mathrm{BN}$ analysis is possible when there is a causal relationship between two nodes. For EM risk analysis, the risk factors listed in Table 1 can be considered as the causal factors. From Table 1, if we consider functional dependence between components as a causal factor to determine the probability of the degree of EMI impact, we get a graph similar to Fig. 2(b). The arcs in Fig. 2 denote the functional dependence between nodes. In Fig 2(b), the power controller $\mathrm{C} 2$ receives the input power supply through the interface cable $\mathrm{C} 5$, making $\mathrm{C} 2$ functionally dependent on $\mathrm{C} 5$.

To demonstrate the use of BN in EM risk analysis, let us consider the sub-graph shown in Fig. 2(b), consisting of node C2, its child nodes (C4 and $\mathrm{C} 6)$ and its parent nodes ( $\mathrm{C} 5$ and $\mathrm{C} 7)$. In this case, in addition to the individual EMI impact on functional performance of C2, EMI impact on the parents of C2 should also be considered. So, if we assign a variable to denote the EM susceptibility of each node, then the conditional probability measure $P(\mathrm{C} 2 \mid \mathrm{pa}(\mathrm{C} 2))$, denoting "the probability of deviation of the safety function performed by node $C 2$ due to EMI, given its functional dependence on its parent nodes $p a(C 2)$ ", can be determined using [7]:

$$
P(\mathrm{C} 2 \mid \mathrm{pa}(\mathrm{C} 2))=P(\mathrm{C} 2 \cap \mathrm{C} 5 \cap \mathrm{C} 7) / P(\mathrm{C} 5 \cap \mathrm{C} 7)
$$

where the terms $P(\mathrm{C} 2 \cap \mathrm{C} 5 \cap \mathrm{C} 7)$ and $P(\mathrm{C} 5 \cap \mathrm{C} 7)$ represent joint probability distributions (JPD) over their arguments.

If all of the nodes in Fig. 2(b) are responsible for a system-level function, then the overall probability for the functional deviation at system-level can be determined from their JPD, which in this example can be determined using the factorization [8]:

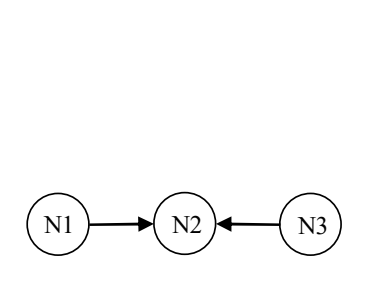

(a)

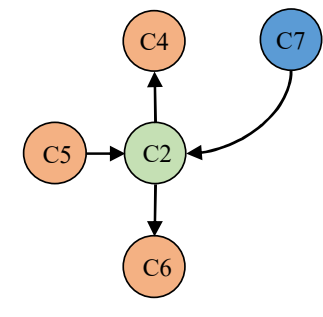

(b)

Fig. 2. (a) An example of a BN; (b) Sub-graph from Fig.1 to represent the dependence/interactions of $\mathrm{C} 2$ with other components of the system in the form of a BN.

$$
\begin{aligned}
& P(\mathrm{C} 2 \cap \mathrm{C} 4 \cap \mathrm{C} 5 \cap \mathrm{C} 6 \cap \mathrm{C} 7)= \\
& P(\mathrm{C} 5) \cdot P(\mathrm{C} 7) \cdot P(\mathrm{C} 2 \mid \mathrm{C} 5 \cap \mathrm{C} 7) \cdot P(\mathrm{C} 4 \mid \mathrm{C} 2) \cdot P(\mathrm{C} 6 \mid \mathrm{C} 2)
\end{aligned}
$$

where $P(\mathrm{C} 5)$ and $P(\mathrm{C} 7)$ are the independent (also called marginal) probability measures for the functional deviation due to EMI in nodes $\mathrm{C} 5$ and $\mathrm{C} 7$ (respectively), $P(\mathrm{C} 5 \cap \mathrm{C} 7)$ is the JPD for $\mathrm{C} 5$ and $\mathrm{C} 7$, and the terms $P(\mathrm{C} 4 \mid \mathrm{C} 2)$ and $P(\mathrm{C} 6 \mid \mathrm{C} 2)$ are the conditional probabilities for the functional deviation due to EMI in nodes C4 and C6, respectively, given $\mathrm{C} 2$.

The application of a BN to determine a probability measure for the vulnerability of a building installation to intentional EMI (i.e. a cybersecurity issue) is described in [9]-[10], based on EM topolgy, fault tree analysis and event tree analysis. However, if there is a feedback loop (such as $\mathrm{C} 2 \rightarrow \mathrm{C} 4 \rightarrow \mathrm{C} 1 \rightarrow \mathrm{C} 7 \rightarrow \mathrm{C} 2$ in Fig. 1), Bayesian inference cannot be applied, as BNs are invalid for cyclic graphs. However, other graphical methods, such as the Markov random fields described below, can be employed when cyclic loops are present.

\section{B. Markov Random Fields}

A Markov random field (MRF), also called as a Markov network or a Gibbs distribution, is analogous to a $\mathrm{BN}$, except that it is an undirected graph. The nodes in an MRF graph have a simple linked $(u-v)$ relationship with all their connected nodes (as shown in Fig. 3 ), instead of the parent-child $(u \rightarrow v)$ relationship of BN. The advantage of MRF over BN for a performing a system level risk analysis is that, with MRF, cyclic graphs can be accommodated.

In MRF, the factorisation of probability distribution for the undirected graphical model is according to structures called cliques of the graph [8]. A clique $q$ is a subset of the nodes in a graph that are fully connected (i.e. all nodes in the subset are linked to each other), and the set of maximal cliques, $Q$ contains the cliques with the highest number of nodes.

A sub-graph of the MRF representing the case study (i.e. the graph of Fig. 1 but with undirected arcs replacing the directed arrows) is shown in Fig. 3. This has $N=4$ nodes and four maximal cliques (all 2-node cliques only). Each of the cliques is associated with a compatibility function, $\psi_{q}:\left(\bigotimes_{n \in q} x_{n}\right) \rightarrow \mathbb{R}+$, where $\left(\bigotimes_{n \in q} x_{n}\right)$ denotes the Cartesian product of the state spaces $x_{n}$, corresponding to each variable of node $n \in q$ and $\rightarrow \mathbb{R}+$ indicates that the results are positive real numbers. The compatibility functions are arbitrary non-negative functions (commonly exponentials) that define the interaction between the variables within the clique. If each of the $N$ node variables has $K$ states, then the JPD for Fig. 3 may be obtained from:

$$
P(\mathrm{C} 1 \cap \mathrm{C} 2 \cap \mathrm{C} 4 \cap \mathrm{C} 7)=\prod_{q \in Q} \psi_{q}\left(x_{n \in q}\right) / \sum_{i=1}^{K^{N}} \prod_{q \in Q} \psi_{q}\left(x_{n \in q}\right)
$$

The compatibility functions $\psi_{Q}$ need not have any obvious or

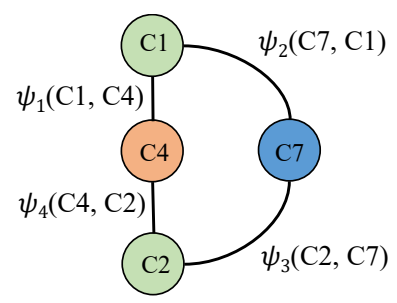

Fig. 3. The looped sub-graph from the case study model, represented here using MRF. 
Accepted version: L. Devaraj, A. R. Ruddle and A. P. Duffy, "Electromagnetic Risk Analysis for EMI Impact on Functional Safety with Probabilistic Graphical Models and Fuzzy Logic," in IEEE Letters on Electromagnetic Compatibility Practice and Applications, doi: 10.1109/LEMCPA.2020.3017483. (C) 2020 IEEE https://ieeexplore.iee.org/document/9171307

direct relation to marginal or conditional probabilities [8], as the child-parent sets in BN have. However, without the causal relationship, the risk factors such as functional dependence cannot be visualized directly from the graphical representation.

\section{Fuzzy Set Theory}

The application of fuzzy logic or fuzzy sets is widely reported (e.g. [11]) as it is very useful tool for risk assessment and decision making. This method is used when the system knowledge/response involves uncertainty, imprecise values/boundaries, scarce and limited datasets. Unlike the two methods discussed above, a fuzzy variable is associated with an uncertain possibility distribution instead of a probability distribution. So, this method is advantageous as the system knowledge for many complex systems may be represented in a wide range of formats and is often imprecise.

To illustrate the concept of fuzzy logic in EM risk analysis terms, consider the proposition "the probability $C 2$ being affected by EMI is most likely to be up to $20 \%$, but certainly not more than $60 \% "$. This can be described by a membership function of the form:

$$
\mu(u)=1-S(u ; \alpha, \beta, \gamma)
$$

where $u$ represents the approximate probability value of $\mathrm{C} 2$ being affected by EMI and the function $S(u ; \alpha, \beta, \gamma)$ is defined [12] by:

$$
S(u ; \alpha, \beta, \gamma)=\left\{\begin{array}{cl}
0 & \text { for } u \leq \alpha \\
2\left[\frac{\{u-\alpha\}}{\{\gamma-\alpha\}}\right]^{2} & \text { for } \alpha \leq u \leq \beta \\
1-2\left[\frac{\{(u-\gamma\}}{\{\gamma-\alpha\}}\right]^{2} & \text { for } \beta \leq u \leq \gamma \\
1 & \text { for } u \geq \gamma
\end{array}\right.
$$

The parameters $\alpha$ and $\gamma$ are constants assigned to define the lower and upper limits (respectively) of the fuzzy distribution (to be estimated by the domain expert), and $\beta$ defines a crossover point, set here such that $S(u ; \alpha, \beta, \gamma)=0.5$. Setting $\alpha=0.2, \beta=0.4$ and $\gamma=0.6$, the function $\mu(u)$ has the form illustrated by the blue line shown in Fig. 4, which reflects the proposition above.

Although in the example above the membership function is illustrated using a particular form, other functions (such as triangular, trapezoidal, Gaussian etc.) could be used to represent different propositions expressed in other formats. For example, the triangular function shown by the orange line in Fig. 4 could be used to represent a proposition of the form "the probability $C 2$ being affected by EMI is most likely to be around $40 \%$, but certainly not less than $20 \%$ and

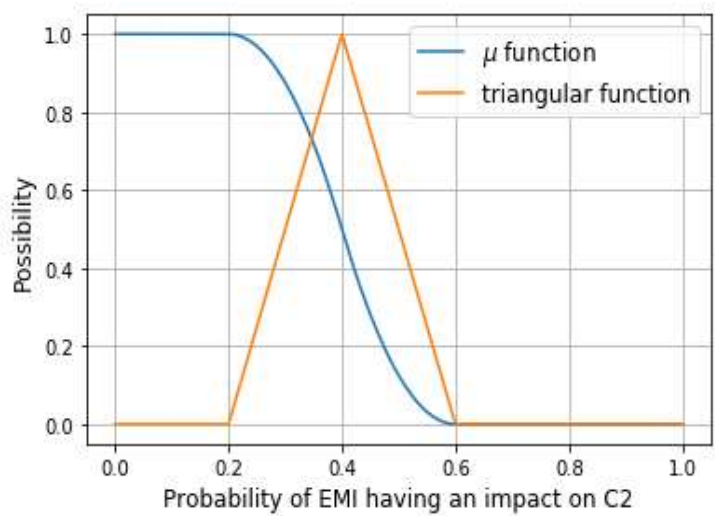

Fig. 4. Possibility measure for the probability of $\mathrm{C} 2$ being affected by EMI, based on membership functions reflecting imprecise domain knowledge. not more than 60\%". Alternatively, a Gaussian function could be used for propositions that do not specify an interval with hard limits.

A possible method for risk analysis by using fuzzy set theory and expert knowledge with FTA through BN modelling is discussed in [13]. A similar approach, combining two or more methods with the graphical model, could also be employed to enable EM risk analysis for functional safety.

\section{CONCLUSION}

In general, reducing the EMI risks associated with safety-related malfunctions is done by implementing safety mechanisms, and by applying techniques and measures suggested by guides and standards, such as shielding, filtering, grounding etc. However, adequate confidence for the overall safety risk to be acceptable, can be achieved only by performing a comprehensive risk analysis. The graphical model introduced in this report is aimed to demonstrate the risk due to EMD on the system, by associating EMI impact on the functions and interactions between components of the system. Combinations of the methods discussed in this paper with the graphical model are being further developed for the estimation of the risk parameters associated with identified hazards. As this graphical approach also enables a better system visualization, it also has the capability to support the entire risk management process.

As future work, to demonstrate and validate the techniques discussed in this paper for vehicle-level EM risk estimation, methods for estimating the risk parameters (probability and degree of EMI impact) will be determined using one or more suitable methods: These may include system knowledge and domain expertise to support the selection of risk parameters at the time of analysis, as well as relevant data (possibly statistical) obtained from experiments or EM simulations.

\section{REFERENCES}

[1] Deutschmann, B, Winkler G, Kastner, P (2018), "Impact of electromagnetic interference on the functional safety of smart power devices for automotive applications," in Elektrotechnik und Informationstechnik, Vol. 135, pp. 352-359.

[2] UNECE Regulation 10 (2014), "Uniform provisions concerning the type approval of vehicles with regard to electromagnetic compatibility", Addendum 9, Rev. 5.

[3] Armstrong, K (2006), "Why EMC testing is inadequate for functional safety - and what should be done instead," Proc $1^{\text {st }}$ IET Int. Conf. System Safety, London, UK, pp. 179-183.

[4] Ruddle, A R, Martin, A J M (2019), "Adapting automotive EMC to meet the needs of the $21^{\text {st }}$ century," IEEE EMC Mag., vol. 8, no. 3, pp. 75-85.

[5] Devaraj, L, Ruddle, A R, Duffy, A P (2020), "System level risk analysis for immunity in automotive functional safety analyses," Proc. EMC Europe 2020, to be published.

[6] BS ISO 26262 (2018), "Road vehicles — Functional safety," British Standards Institute.

[7] Kolmogorov, A (1956), Foundations of the Theory of Probability, Chelsea.

[8] Wainwright M J, Jordan M I (2008), "Graphical models, exponential families, and variational inference," in Foundations and Trends in Machine Learning, vol. 1, Nos. 1-2, pp. 1-13

[9] Mao C, Canavero, F (2016), "System-level vulnerability assessment for EME: from fault tree analysis to Bayesian networks-Part I: Methodology framework," IEEE Trans. EMC, vol. 58, no. 1, pp. 180-187.

[10] Mao C, Canavero, F, Cui, Z, Sun, D (2016), "System-level vulnerability assessment for EME: from fault tree analysis to Bayesian networks-Part II: Illustration to microcontroller system," IEEE Trans. EMC, vol. 58, no. 1, pp. 188-196.

[11] Peikert T, Garbe H, Potthast S (2017), "Fuzzy-based risk analysis for IT-systems and their infrastructure," IEEE Trans. EMC, vol. 59, no. 4, pp. 1294-1301.

[12] Zadeh L A (1999), "Fuzzy sets as a basis for a theory of possibility," in Fuzzy Sets and Systems, 100 Supplement, North-Holland, pp. 9-34.

[13] Yazdi M, Kabir S (2017), "A fuzzy Bayesian network approach for risk analysis in process industries," Process Safety and Env. Prot., vol. 111, pp. 507-519. 